\title{
REMARKS ON A THEOREM OF KORANYI AND MALLIAVIN ON THE SIEGEL UPPER HALF PLANE OF RANK TWO
}

\author{
KENNETH D. JOHNSON
}

\begin{abstract}
In [5], A. Koranyi and P. Malliavin showed that bounded functions on the Siegel upper half plane of rank two which satisfied two special elliptic differential equations were characterized by their values on the Bergman-Silov boundary. In this paper a simple proof of this theorem is given.
\end{abstract}

1. Introduction. The Siegel upper half plane of rank $2, H_{2}$ is defined as the space of $2 \times 2$-matrices $Z$ with complex entries such that $Z=Z^{\prime}$ (' denotes transpose) and $\operatorname{Im} Z>0$ equipped with the metric

$$
d s^{2}=\operatorname{tr}\left((Z-\bar{Z})^{-1} d \bar{Z}(Z-\bar{Z})^{-1} d Z\right) .
$$

The Laplace operator associated to this metric is easily seen to be

$$
\Delta_{1}=\operatorname{tr}\left((Z-\bar{Z}) \partial_{\bar{Z}}(Z-\bar{Z}) \partial_{z}\right)
$$

when for $A=A^{\prime}, \partial_{A}=\left(\partial_{i j}\right)$ with $\partial_{i j}=\frac{1}{2}\left(1+\delta_{i j}\right) \partial / \partial a_{i j}$ and $\partial_{\bar{Z}}$ does not differentiate the entries of $Z-\bar{Z}$. Also, of interest to us is the operator

$$
\Delta_{2}=\operatorname{tr}\left(\partial_{\bar{Z}}(Z-\bar{Z}) \partial_{Z}\right) \text {. }
$$

The Bergman-Šlov boundary $\mathrm{B}_{2}$ of $\mathrm{H}_{2}$ contains the $2 \times 2$ real symmetric matrices $S_{2}$ as an open dense subset and the Poisson kernel of $\mathrm{H}_{2}$ with respect to $B_{2}$ is the function $P_{0}: H_{2} \times B_{2} \rightarrow \mathbf{R}^{+}$such that

$$
P_{0}(Z, U)=c\left(\frac{\operatorname{det}(Z-\bar{Z})}{|\operatorname{det}(Z-U)|^{2}}\right)^{3 / 2} \quad\left(Z \in H_{2}, U \in S_{2}\right) \text {. }
$$

In [5], A. Koranyi and P. Malliavin showed that if $f \in L^{\infty}\left(H_{2}\right)$ and $\Delta_{1} f=\Delta_{2} f=0$

$$
f(Z)=\int_{S_{2}} P(Z, U) F(U) d U
$$

for some $F \in L^{\infty}\left(S_{2}\right)$. In this paper, we give a new proof of this result which uses the techniques found in [4] rather than diffusion processes. We first introduce the group of symplectic transformations.

Received by the editors November 5, 1976.

AMS (MOS) subject classifications (1970). Primary 22E30, 32M15; Secondary 43A85, 53C35.

Key words and phrases. Poisson kernel, Bergman-Šilov boundary, Furstenberg boundary, symplectic transformations. 
If $\operatorname{Sp}(2, \mathbf{R})$ is the group of $4 \times 4$-matrices $X$ with real entries which have the property that $X J=J X^{\prime}$ for

$$
J=\left(\begin{array}{cc}
0 & I_{2} \\
-I_{2} & 0
\end{array}\right) \quad\left(I_{2} \text { is } 2 \times 2 \text { identity matrix }\right),
$$

$S p(2, \mathbf{R})$ acts transitively on $H_{2}$ as follows. For $Z \in H_{2}$ and $g=\left(\begin{array}{cc}A & B \\ C & D\end{array}\right) \in$ $\operatorname{Sp}(2, \mathbf{R})(A, B, C, D 2 \times 2$-matrices $)$ then

$$
g(Z)=(A Z+B)(C Z+D)^{-1} \text {. }
$$

Since $\mathrm{Sp}(2, \mathbf{R})$ acts on $\mathrm{H}_{2}$ it also acts on functions defined on $\mathrm{H}_{2}$. If $f$ : $\mathrm{H}_{2} \rightarrow \mathrm{C}$,

$$
\operatorname{Lg} f(Z)=f\left(g^{-1}(Z)\right) \quad(g \in \operatorname{Sp}(2, \mathbf{R})) .
$$

Then for $g \in S p(2, \mathbf{R}), \Delta_{1} L g f=L g \Delta_{1} f$ and although $\Delta_{2} L g f \neq L g \Delta_{2} f$ in general it is true that if

$$
g=\left(\begin{array}{cc}
I & X \\
0 & I
\end{array}\right) \quad\left(X=X^{\prime}\right) \quad \text { or } \quad g=\left(\begin{array}{ll}
A & 0 \\
0 & A
\end{array}\right) \quad(A \in 0(2))
$$

$\Delta_{2} \operatorname{Lg} f=\operatorname{Lg} \Delta_{2} f$ and if

$$
g=\left(\begin{array}{cc}
a I_{2} & 0 \\
0 & a^{-1} I_{2}
\end{array}\right) \quad(a>0)
$$

$L g \Delta_{2} f=a^{-2} \Delta_{2} L g f$. Thus, if $K\left(H_{2}\right)=\left\{f \in L^{\infty}\left(H_{2}\right): \Delta_{1} f=\Delta_{2} f=0\right\}, L g$ : $K\left(\mathrm{H}_{2}\right) \rightarrow K\left(\mathrm{H}_{2}\right)$ for

$$
g=\left(\begin{array}{cc}
a A & a A X \\
0 & a^{-1} A
\end{array}\right) \quad\left(X=X^{\prime}, A \in 0(Z), a>0\right) .
$$

Here we point out that Koranyi and Malliavin [5] in fact consider the operator $\Delta_{2}^{1}=c(\operatorname{tr}(Z-\bar{Z})) \Delta_{2}$ and then $L g \Delta_{2}^{1} f=\Delta_{2}^{1} L g f$ for $g$ as above and $K\left(H_{2}\right)=\left\{f \in L^{\infty}\left(H_{2}\right): \Delta_{1} f=\Delta_{2}^{1} f=0\right\}$.

If $f \in K\left(\mathrm{H}_{2}\right)$ it follows from Furstenberg [1] that

$$
f(Z)=\int_{B} P(Z, b) F(b) d b
$$

for some $F \in L^{\infty}(B)$ when $B$ is the Furstenberg boundary of $H_{2}$ and $P$ : $H_{2} \times B \rightarrow \mathbf{R}^{+}$a Poisson kernel and $d b$ is the $K=O(4) \cap S p(2, \mathbf{R})$-invariant measure on $B$ normalized so that $\int_{B} d b=1$. After observing that $B=K / M$ where $M$ is the diagonal matrices in $K$, we have that $P: G \times K \rightarrow \mathbf{R}^{+}$where

$$
P(g, k)=c\left\|g^{-1} k \vec{e}_{3}\right\|^{-2}\left\|g^{-1} k \vec{e}_{3} \wedge \vec{e}_{4}\right\|^{-2} \text {. }
$$

$\vec{e}_{i}$ is column vector with a 1 in the $i$ th position 0 's elsewhere and \|\| is the norm on $\Lambda \mathbf{R}^{n}$.

2. Reduction to a special case. Suppose now that $f \in K\left(H_{2}\right)$ and

$$
f(Z)=\int_{B} P(Z, b) F(b) d b \quad\left(F \in L^{\infty}(B)\right)
$$


Let $K_{0}$ be the group of all matrices $g$ when

$$
g=\left(\begin{array}{cc}
A & 0 \\
0 & A
\end{array}\right) \quad(A \in 0(2))
$$

and define for $\alpha \in C^{\infty}\left(K_{0}\right)$

$$
\alpha{ }_{K_{0}} f(Z)=\int_{K_{0}} \alpha(k) f\left(k^{-1}(Z)\right) d k .
$$

Then

$$
\alpha *{ }_{K_{0}} f(Z)=\int_{B} P(Z, b)\left(\alpha *{ }_{K_{0}} F\right)(b) d b .
$$

Let $\hat{K}_{0}$ denote the set of equivalence classes of finite dimensional irreducible representations of $K_{0}$ and identify $\tau \in \hat{K}_{0}$ with its members. Then if we set $\chi_{\tau}(k)=(\operatorname{deg} \tau) \operatorname{tr} \tau(k)$ we have

(1) $\sum_{\tau \in \hat{K}_{0}} \chi_{\tau}{ }^{*}{ }_{K_{0}} f$ converges to $f$ in $C^{\infty}\left(H_{2}\right)$;

(2) $\Sigma_{\tau \in \hat{K}_{0}} \chi_{\tau}^{*}{ }^{*} F$ converges to $F$ in $L^{2}(B)$.

Since $B_{2}=K / K_{0}$ to prove our result it suffices to show that each $\chi_{\tau}{ }^{*}{ }_{K_{0}} F$ is a function on $B_{2}$ or $S_{2}$.

Again let $\bar{N}$ be the group of all matrices $g$ where

$$
g=\left(\begin{array}{cc}
I & X \\
0 & I
\end{array}\right) \quad\left(X=X^{\prime}\right)
$$

Select $\left\{\phi_{n}: n \geqslant 0\right\} C_{c}^{\infty}(\bar{N})$ so that $\phi_{n} \rightarrow \delta$. Then $\phi_{n}{ }^{*} \bar{N} f \rightarrow f$ as $n \rightarrow \infty$ in $\mathrm{C}^{\infty}\left(\mathrm{H}_{2}\right)$ and we see that

$$
\left(\phi_{n} *{ }_{\bar{N}} f\right)(Z)=\int_{B} P(Z, b)\left(\phi_{n}{ }^{*}{ }_{N} F\right)(b) d b .
$$

By observing that $\bar{N} \cong S_{2}$ and selecting our $\phi_{n}$ so that $\phi_{n}\left(A X A^{-1}\right)=\phi_{n}(X)$ for $A \in O(2), X \in S_{2}$ we have that

$$
\phi_{n} * \bar{N} \chi_{\tau}{ }^{*}{ }_{K_{0}} f=\chi_{\tau}{ }^{*}{ }_{K_{0}} \phi_{n} * \bar{N} f
$$

Writing $B=G / P$ for $P$ some subgroup of $G$ we have that $\bar{N} K_{0} P$ is open and dense in $B$ and $B \sim \bar{N} K_{0} P$ has measure 0 . Thus almost everywhere we may express $F$ as an $L^{\infty}$ function on $S_{2} \times K_{0} \cong S_{2} \times S^{1}$

$$
F(X ; \theta) \quad\left(X \times e^{i \theta} \in S_{2} \times S^{1}\right) \text {. }
$$

Convolving with a $\phi_{n}$ we have that if $D$ is any differential operator with constant coefficients in the $X$-variables $\sup _{X, \theta}|D F(X, \theta)|<\infty$. After writing

$$
X=\left(\begin{array}{cc}
x_{1}+x_{2} & x_{3} \\
x_{3} & x_{1}-x_{2}
\end{array}\right) \quad\left(x_{1}, x_{2}, x_{3} \text { real }\right)
$$

and setting $\left(x_{2}, x_{3}\right)=(r, 2 \phi)$ (polar coordinates) we have that $F$ is a function in the variables $\left(x_{1}, r, \phi ; \theta\right)$. Convolving $F$ over $K_{0}$ with some $\chi_{\tau}$ we have that

$$
F\left(x_{1}, r, 2 \phi+2 \psi ; \theta+\psi\right)=e^{2 / \psi} F\left(x_{1}, r, \phi ; \theta\right)
$$

for some integer $l$ and hence

$$
F\left(x_{1}, r, 2 \phi ; \theta\right)=e^{2 l \phi} F\left(x_{1}, r, 0, \theta-\phi\right) .
$$


Our result will follow if we can show that $F$ is independent of $\theta$.

3. Main result. For $Z \in \mathrm{H}_{2}$ it will be convenient to use the coordinates

$$
Z=\left(\begin{array}{cc}
z_{1}+z_{2} & z_{3} \\
z_{3} & z_{1}-z_{2}
\end{array}\right)
$$

as was done in [5]. Then

$$
\Delta_{2}^{1}=c\left(y_{1}^{2} \sum_{i=1}^{3} \bar{\partial}_{i} \partial_{i}+\sum_{i=2}^{3} y_{1} y_{i}\left(\bar{\partial}_{i} \partial_{1}+\bar{\partial}_{1} \partial_{i}\right)\right)={ }^{0} \Delta_{2}+\Delta_{2}^{0}
$$

where

$$
{ }^{0} \Delta_{2}=\frac{c}{4}\left(y_{1}^{2} \sum_{i=1}^{3} \frac{\partial^{2}}{\partial x_{i}^{2}}+\sum_{i=2}^{3} 2 y_{1} y_{i} \frac{\partial^{2}}{\partial x_{1} \partial x_{i}}\right)
$$

and

$$
\Delta_{2}^{0}=\frac{c}{4}\left(y_{1}^{2} \sum_{i=1}^{3} \frac{\partial^{2}}{\partial y_{i}^{2}}+\sum_{i=2}^{3} 2 y_{1} y_{i} \frac{\partial^{2}}{\partial y_{1} \partial y_{i}}\right) .
$$

Suppose now that $f \in K\left(H_{2}\right)$ and

$$
f(Z)=\int_{S_{2} \times S^{1}} P(Z, X ; \theta) F(X ; \theta) d x d \theta
$$

where $F\left(x_{1} ; r, 2 \phi ; \theta\right)=e^{i l \phi} F\left(x_{1} ; r, 0 ; \theta-\phi\right)$ and $\left|\sup _{x, \theta} D F(X ; \theta)\right|<\infty$ for $D$ any differential operator in the $x$-variables with constant coefficients.

Let

$$
H=\left(\begin{array}{cccc}
-a_{1} & 0 & 0 & 0 \\
0 & -a_{2} & 0 & 0 \\
0 & 0 & a_{1} & 0 \\
0 & 0 & 0 & a_{2}
\end{array}\right) \quad\left(a_{1}>a_{2}>0\right)
$$

and set $f_{H}(g)=\lim _{\tau \rightarrow \infty} f(g$ expt $H)$. Then we have the following lemma.

LEMMA 3.1. $f_{H}=F$ almost everywhere and $\left({ }^{0} \Delta_{2} f\right)_{H}=0$ on $S^{2} \times S^{1}$.

Proof. That $f_{H}=F$ almost everywhere is proved in Helgason and Koranyi [3]. That $\left({ }^{0} \Delta_{2} f\right)_{H}=0$ follows first by differentiating under the integral to obtain

$$
\frac{\partial^{2}}{\partial x_{i} \partial x_{j}} f(Z)=\int_{S^{\prime}} \int_{S_{2}} \frac{\partial^{2}}{\partial x_{i} \partial x_{j}} P(Z ; U ; \theta) F(U ; \theta) d U d \theta
$$

and by the conditions on $F$ to obtain

$$
\frac{\partial^{2}}{\partial x_{i} \partial x_{j}} f(Z)=\int_{S^{1}} \int_{S_{2}} P(Z ; U ; \theta) \frac{\partial^{2}}{\partial u_{i} \partial u_{j}} F(U ; \theta) d U d \theta \text {. }
$$

Our result now follows by combining the first statement of the lemma with the property that $y_{i} \rightarrow 0$ for each $i$. 
We are now in a position to obtain our main result. To do this we must examine the equation $\left(\Delta_{2}^{0} f\right)_{H}=0$. A few more preliminaries are necessary to accomplish this.

If $Z=X+i Y \in H_{2}, Z=g_{x} g_{Y} i$ for

$$
g_{x}=\left(\begin{array}{cc}
I & X \\
0 & I
\end{array}\right) \text { and } g_{Y}=\left(\begin{array}{cc}
Y^{1 / 2} & 0 \\
0 & Y^{-1 / 2}
\end{array}\right)
$$

but $Z=g_{x} g_{T} i$ for some

$$
g_{T}=\left(\begin{array}{cc}
T & 0 \\
0 & T^{\prime}
\end{array}\right) \quad\left(T=\left(\begin{array}{cc}
x & y z \\
0 & z
\end{array}\right) \quad x, y, z \text { real }\right)
$$

An easy computation yields

$$
\begin{gathered}
y_{1}=\frac{x^{2}+\left(y^{2}+1\right) z^{2}}{2}, \quad y_{2}=\frac{x^{2}+\left(1-y^{2}\right) x^{2}}{2}, \quad y_{3}=y z^{2}, \\
\frac{\partial}{\partial y_{1}}=\frac{1+y^{2}}{2 x} \frac{\partial}{\partial x}-\frac{y}{z^{2}} \frac{\partial}{\partial y}+\frac{1}{2 z} \frac{\partial}{\partial z}, \\
\frac{\partial}{\partial y_{2}}=\frac{1-y^{2}}{2 x} \frac{\partial}{\partial x}+\frac{y}{z^{2}} \frac{\partial}{\partial y}-\frac{1}{2 z} \frac{\partial}{0 z}, \\
\frac{\partial}{\partial y_{3}}=\frac{-y}{x} \frac{\partial}{\partial x}+\frac{1}{z^{2}} \frac{\partial}{\partial y} .
\end{gathered}
$$

Modifying the arguments of Lemma 2.9 of [4] to see that $(\partial f / \partial x)_{H}=$ $(\partial f / \partial z)_{H}=0$ we obtain

LEMMA 3.2. $\left(\Delta_{2}^{0} f\right)_{H}=c d / d y\left(\left(1+y^{2} / 2\right)^{2} d / d y F\left(X ; \tan ^{-1} y\right)\right)=0$.

Thus we obtain setting $\theta=\tan ^{-1} y$ that $d / d \theta\left(\sec ^{2} \theta d / d \theta\right) F(X ; \theta)=0$ and solving we have

$$
F\left(x_{1} ; r, 2 \phi ; \theta\right)=F_{1}\left(x_{1} ; r, 2 \phi\right)+F_{2}\left(x_{1} ; r, 2 \phi ; 0\right)(\sin 2 \theta+2 \theta)
$$

when $F_{1}$ is independent of $\theta$. Recall that

$$
F\left(x_{1} ; r, 2 \phi ; \theta\right)=e^{i l \phi} F\left(x_{1} ; r, 0 ; \theta-\phi\right) .
$$

Then

$$
\begin{aligned}
e^{i l \phi} F\left(x_{1} ; r,\right. & 0, \theta-\phi) \\
& =e^{i l \phi}\left(F_{1}\left(x_{1} ; r, 0\right)+F_{2}\left(x_{1} ; r, 0 ; 0\right)(\sin 2(\theta-\phi)+(\theta-\phi))\right) .
\end{aligned}
$$

As $F\left(x_{1} ; r, 2 \phi ; 0\right)=F_{1}\left(x_{1} ; r, 2 \phi\right)$ we have

$$
F_{1}\left(x_{1} ; r, 2 \phi\right)=e^{i l \phi}\left(F_{1}\left(x_{1} ; r, 0\right)-F_{2}\left(x_{1} ; r, 0 ; 0\right)(\sin 2 \phi+2 \phi)\right)
$$

and so

$$
\begin{aligned}
& e^{i l \phi}\left(F_{1}\left(x_{1} ; r, 0\right)+F_{2}\left(x_{1} ; r, 0 ; 0\right)(\sin 2(\theta-\phi)+2(\theta-\phi))\right) \\
&= e^{i l \phi}\left(F_{1}\left(x_{1}, r, 0\right)-F_{2}\left(x_{1} ; r, 0 ; 0\right)(\sin 2 \phi+2 \phi)\right) \\
&+F_{2}\left(x_{1} ; r, 2 \phi ; 0\right)(\sin 2 \theta+2 \theta) .
\end{aligned}
$$


Thus

$$
\begin{aligned}
& F_{2}\left(x_{1} ; r, 2 \phi ; 0\right)(\sin 2 \theta+2 \theta) \\
& \quad=F_{2}\left(x_{i} r, 0 ; 0\right) e^{i l \phi}((\sin 2 \phi+2 \phi)+(\sin 2(\theta-\phi)+2(\theta-\phi)))
\end{aligned}
$$

and dividing by $\sin 2 \theta+2 \theta$ we see that the right-hand side of the equation is not independent of $\theta$ unless $F_{2}=0$ and finally we obtain the lemma.

LEMMA 3.3. $F$ is independent of $\theta$.

REMARK. That $F_{2}=0$ also follows immediately from the boundedness of $F$. Finally we summarize.

Theorem 3.4 (Koranyi and Malliavin [5]). If $f \in K\left(H_{2}\right)$

$$
f(Z)=\int_{B_{2}} P_{0}(Z, b) F(b) d b
$$

for some $F \in L^{\infty}\left(B_{2}\right)$.

\section{REFERENCES}

1. H. Furstenberg, A Poisson formula for semi-simple Lie groups, Ann. of Math. 77 (1963), 335-386.

2. L. K. Hua, Harmonic analysis of functions of several complex variables in the classical domains, Transl. Math. Monographs, vol. 6, Amer. Math. Soc., Providence, R. I., 1963.

3. S. Helgason and A. Koranyi, A Fatou-type theorem for harmonic functions on symmetric spaces, Bull. Amer. Math. Soc. 74 (1968), 258-263.

4. K. D. Johnson, Differential equations and the Bergman-Silov boundary on the Siegel upper halfplane, Ark. Mat. (to appear).

5. A. Koranyi and P. Malliavin, Poisson formula and compound diffusion associated to an overdetermined elliptic system on the Siegel halfplane of rank two, Acta Math. 134 (1975), 185-209.

Department of Mathematics, Swain Hall-East, Indiana University, Bloomington, INDIANA 47401

Current address: Department of Mathematics, University of Georgia, Athens, Georgia 30602 